\title{
Multichannel time-to-digital converters with automatic calibration in Xilinx Zynq-7000 FPGA devices
}

\author{
Yu Wang, Wujun Xie, Haochang Chen, and David Day-Uei Li
}

\begin{abstract}
This paper proposes a weighted histogram calibration method and an automatic calibration architecture to implement high-linearity time-to-digital converters (TDCs) in low-cost ARM-based System-onChips (SoCs). The proposed method significantly reduces the nonlinearity introduced by nonuniform bins. It offers automatic calibration without manual interventions using ARM processors. Besides, our design is cost-effective in hardware consumption. We implemented and evaluated a 16-channel TDC system in a low-cost Zynq-7000 ARMbased SoC, in which the programable logic is equivalent to a $28 \mathrm{~nm}$ Artix-7 FPGA. The proposed TDC offers a resolution of 9.83 ps with good uniformity, achieving an averaged $D N L_{p k-p k}$ of $0.38 L S B$, and an averaged $I N L_{p k-p k}$ of 0.63 LSB.
\end{abstract}

Index Terms-Time-to-digital converter (TDC), carry chains, field-programmable gate array (FPGA).

\section{INTRODUCTION}

$\mathrm{T}$ IME resolved measurements are popular in many research fields, for example, light detection and ranging (LiDAR) for autonomous vehicles [1], [2], 3-D reconstruction [3], [4], surveying [5], enhanced Gigabit Ethernet [6], temperature sensing [7], quantum communications [8], [9], particle physics [10], space sciences [11], true random number generation [12], Raman spectroscopy [13], and medical physics such as positron emission tomography (PET) [14], optical reflectance spectroscopy [15], and fluorescence lifetime imaging microscopy (FLIM) [16], [17]. Time-to-digital converters (TDCs) are simply high-precision stopwatches, converting the time interval (TI) between two events into a digital code. They are gaining attention both in scientific instruments and industrial applications [18]-[21].

A growing research trend is to improve the TDC resolution (also called the least significant bit, LSB; it is the minimum measured TI). However, linearity and precision are also important parameters in TDC designs and determine the TDC system's accuracy. TDCs' nonlinearity can be characterized by differential nonlinearity (DNL) and integral nonlinearity (INL). They are defined as Eqs (1) and (2), where $W[k]$ is the $k$-th

Manuscript received April 19, 2021, accepted August 29, 2021. (Yu Wang and Wujun Xie contributed equally to this work.) (Corresponding author: David Day-Uei Li)

Y. Wang, W. Xie, and D. D.-U. Li are with the Faculty of Engineering, University of Strathclyde, Glasgow, G4 ORE, U.K., (e-mail: bin's bin-width, and $Q$ is the averaged bin-width. DNL and INL can be evaluated by code density tests [22].

$$
\begin{gathered}
D N L[k]=\frac{(W[k]-Q)}{Q}, \\
I N L[k]=\sum_{n=0}^{k} D N L[n],
\end{gathered}
$$

The precision $(\sigma)$ shows the repeatability of measurements and can be assessed by time interval tests:

$$
\sigma^{2}=\frac{1}{N-1} \sum_{i=1}^{N}\left(x_{i}-\mu\right),
$$

where $x_{i}$ is the $i$-th output's bin number and $\mu$ is the averaged value of $N$ measurements.

TDCs can be realized in application-specific integrated circuits (ASICs) and field-programmable gate arrays (FPGAs). With recent advances in complementary metal-oxidesemiconductor (CMOS) technologies, both ASIC-TDCs and FPGA-TDCs can achieve picosecond resolutions. Compared with ASIC-TDCs, FPGA-TDCs are cheaper and have shorter developing cycles. However, due to large nonlinearity and the lack of automatic calibration methods, there are only a few commercial FPGA-TDCs [23]. On the contrary, ASIC-TDCs dominate commercial time-correlated single-photon counting products [24]-[26], as they show better linearity and precision due to the careful placement and routing strategies.

The tapped delay line (TDL) structure is the mainstream approach to build TDCs in FPGAs because cascaded carrychain modules are common in modern FPGAs, for example, CARRY4 modules in Xilinx 6-series and 7-series FPGAs [27] and CARRY8 modules in UltraScale and other more advanced FPGAs [28]. The TDL-TDC's resolution is determined by the delay module's propagation delay, limited by CMOS processes. Wave union (WU) methods [29], multichain interpolation methods [30], vernier delay lines (VDLs) [31] and delay matrix structures [32] have been proposed to enhance the resolution.

However, due to clock skews and process variations, TDLs are uneven, resulting in large nonlinearity. Look-up table (LUT) based bin-by-bin calibration [33] methods can reduce the INL. Won and Lee proposed the tuned-TDL structure [34] to improve linearity by changing the output pattern of carry-chain

y.wand.100@strath.ac.uk; wuiun.xie@strath.ac.uk; david.li@strath.ac.uk). H. Chen is with the Fraunhofer UK Research Ltd (e-mail: Haochang.Chen@fraunhofer.co.uk) 
modules and suggested a pattern 'SCSC' for Kintex-7 and Virtex-6 devices and 'SCSS' for the Spartan-6 devices. A block random access memory (BRAM) based mixed calibration method [35] improves TDCs' linearity by a two-step calibration, including bin compensation and width calibration. With the mixed calibration method, a 5.0 ps 96-channel TDC was proposed [35], achieving an averaged peak-to-peak DNL $\left(D N L_{p k-p k}\right)=0.27$ LSB and peak-to-peak INL $\left(I N L_{p k-p k}\right)=$ 0.59 LSB, comparable with ASIC-TDCs. The BRAM-based structure is efficient and flexible. A 128-channel resolutionadjustable TDC for LiDAR applications was implemented [36] using BRAM-based mixed-binning methods. However, calibration coefficients stored in BRAMs are pre-calculated on PCs based on code density tests, making it time-consuming, especially for multichannel designs. Due to nonlinearity and time-consuming channel-by-channel or chip-by-chip calibration, FPGA-TDCs require proper calibration algorithms for broad commercial applications that force a trade-off between the number of channels and area occupancy [37], [38]. This work aims to tackle this problem.

The main contributions of this works are:

1) We proposed a single-step weighted histogram calibration method that can ease ultra-wide bin problems caused by bins whose width is less than 5 LSB, which is more efficient than the previously reported mixed calibration method [35].

2) We proposed an automatic calibration (AC) architecture using ARM-based System-on-Chips (SoCs), without manual calibration.

3) We implemented and tested a 16-channel AC-WU TDC in an ARM-based SoC device: the Xilinx Zynq-7000 SoC (Xilinx XC7Z020).

\section{ARCHITECTURE AND DESIGN}

The TDL architecture is the backbone for the proposed TDC, implemented with CARRY4s in a low-cost Xilinx Zynq-7000 SoC (XC7Z020, ZedBoard development board). Fig. 1a is the system architecture of the proposed TDC. The whole system is composed of a programmable logic (PL, equivalent to Artix 7 FPGA) and a processing system (PS, dual-core ARM Cortex-9 inside) [39]. PL is for implementing TDCs, including TDLs, encoders, and calibration modules. Histogramming modules were also implemented in PL to correct biased timestamps caused by background noise and device jitter [36]. PS is for calculating calibration coefficients. The channel selector in PL is for multichannel applications, transferring data between the TDC channels and the ARM core. The advanced eXtensible interface (AXI) is the data bus for communications between PL and PS [40].

To further improve TDC's performance, techniques, like WU methods [29], sub-TDL structures [35] and tuned TDL methods [34], were also applied in our study.

\section{A. Wave Union Method and Sub-TDL Architecture}

The concept of the WU method is shown in Fig. 1b. When a hit signal arrives at the TDC's input port, a look-up table
(LUT)-based WU launcher generates a rising and a falling transitions separately. These two transitions (0-1 and 1-0) propagate along the TDL, and the TDL's outputs are sampled by D-type flip-flops (DFFs) simultaneously in every tap. With two transitions, the TDC conducts two measurements for the same TI in one clock period, and the WU TDC's resolution can be defined as [41]:

$$
L S B_{W U}=\frac{T}{N_{r}+N_{f}}=\frac{L S B_{r} \times L S B_{f}}{L S B_{r}+L S B_{f}}
$$

where $T$ is the period of the sampling clock, $N_{r}$ and $N_{f}$ are bin numbers of the plain TDC with a rising transition and a falling transition, respectively. Several studies indicate that WU is efficient in enhancing the resolution, but it also exacerbates bubble problems (unexpected transitions of logic states, for example, unexpected zeros among a series of ones), causing encoding failures [29], [42], [43]. Moreover, although the bin realignment method can remove bubbles when only one transition exits in the TDL [44], it is difficult to achieve the same result in WU TDCs due to the speed difference between rising and falling transitions [41].

The sub-TDL [35] method (or the decomposition method [45]) can remove bubbles. Mismatches and large clock skews lead to bubble errors [45], [46]. A study [47] reports that the largest clock skew within a clock region is around $19 \mathrm{ps}$ in Xilinx Kintex-7 FPGAs. However, the typical delay time between two neighboring delay cells (see Fig. 1c, highlighted in yellow) in 7-series FPGAs is around $10-15$ ps [48], [49]. Furthermore, the sub-TDL structure elongates time intervals between taps by decomposing sampling taps. For example, in our design, the interval highlighted in blue is four times longer than the interval highlighted in yellow (see Fig. 1c). Therefore, the sub-TDL structure can minimize the impact of clock skews and mismatches and remove bubbles. Meanwhile, interpolations with four sub-TDLs maintain the TDC's resolution. Therefore, the sub-TDL structure can remove bubbles efficiently without trading off the resolution.

\section{B. CARRY4 and Tuned-TDL Architecture}

As shown in Fig. 1c, the TDL is constructed with cascaded CARRY4s, and each CARRY4 contains four cascaded delay elements. Each delay element has two direct outputs named $C$ and $S$ ports, respectively, and neighboring delay elements are connected through internal routing resources [50]. In [34], Won and Lee reported that TDCs' linearity could be improved by changing output patterns. To find the best sampling pattern for Zynq-7000 SoC, we conducted similar experiments. The results are summarized in Table I, indicating that the pattern "SCSC" performs the best linearity in Zynq-7000 SoCs.

Table I.

SAMPLING PATTERNS COMPARISON

\begin{tabular}{c|c|c}
\hline Pattern (LSB = 9.83 LSB) & DNL (LSB) & INL (LSB) \\
\hline CCCC & {$[-0.99,4.32], 5.32$} & {$[-5.44,4.17], 9.61$} \\
SSSS & {$[-0.91,3.26], 4.17$} & {$[-5.32,4.71], 10.03$} \\
CSCC & {$[-0.98,3.25], 4.23$} & {$[-5.29,5.19], 10.48$} \\
CSCS & {$[-0.95,5.07], 6.02$} & {$[-7.40,5.28], 12.68$} \\
SCSS & {$[-0.97,3.26], 4.24$} & {$[-7.18,5.27], 12.45$} \\
SCSC & {$[-\mathbf{0 . 8 9 , 2 . 9 4}], \mathbf{3 . 8 4}$} & {$[-\mathbf{5 . 7 3}, \mathbf{4 . 9 6}], \mathbf{1 0 . 6 9}$} \\
\hline
\end{tabular}




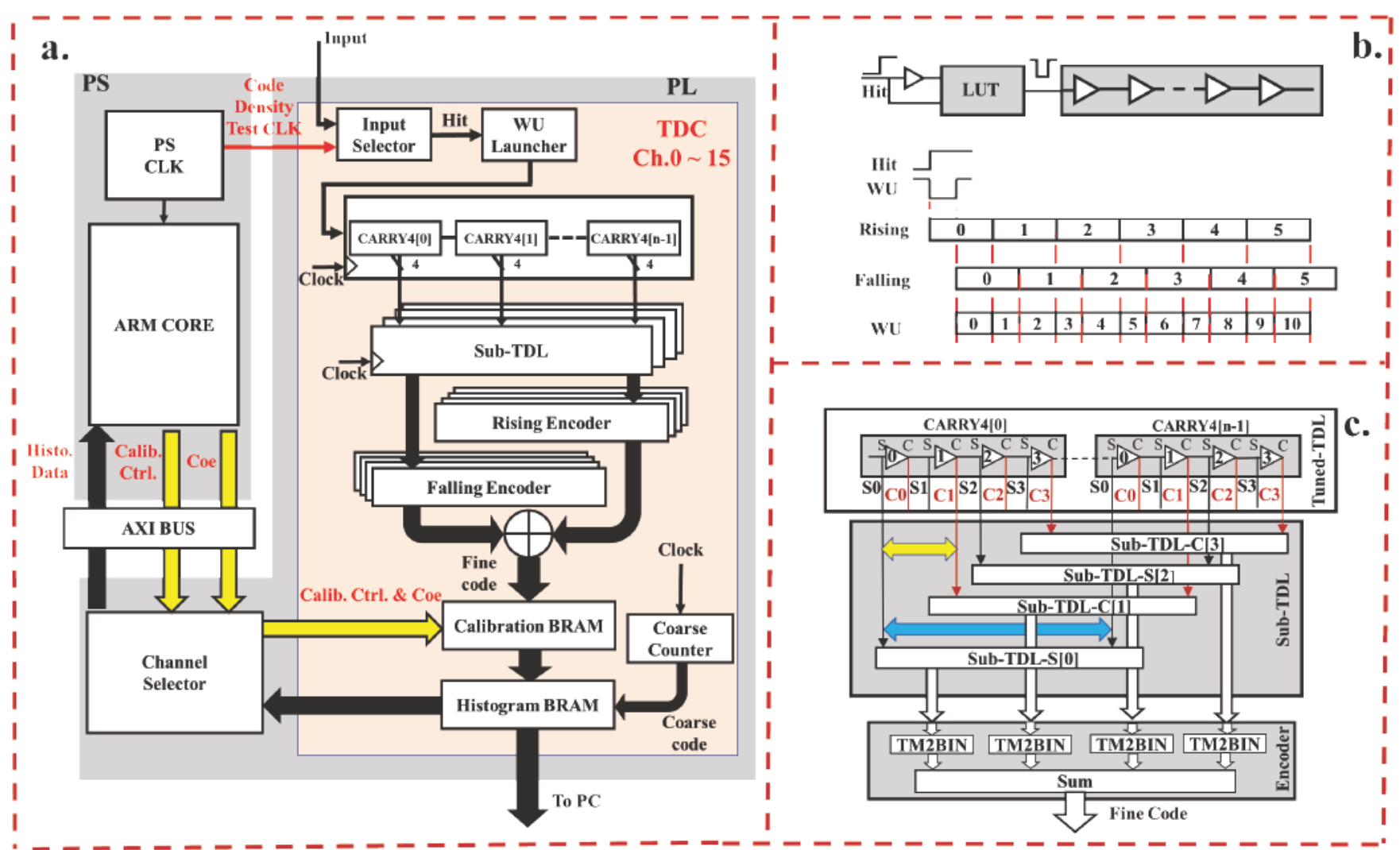

Fig. $\overline{1}$ (a) The block diagram of the proposed TDC system. (b) The concept of the WU method. (c) The block diagram of the tuned-TDL architecture and sub-TDL architecture.

\section{Weighted Histogram Calibration Method}

Although the tunned-TDL method can improve linearity, ultra-wide and ultra-narrow bins still exist and contribute to nonlinearity. To ease ultra-wide bin problems, Chen and $\mathrm{Li}$ proposed the mixed calibration method [35] to achieve excellent linearity, comparable with ASIC-TDCs. The mixed calibration method contains two steps: bin compensation and width calibration. Code density tests are required for each step to calculate correction factors. In the bin compensation, small bins are compensated by large bins by address factors $\left(B C F_{m}\right.$ and $B C F_{c}$ ) (see Fig. 2a). The width calibration performs like the bin-by-bin calibration [33] and further enhances linearity. However, the mixed calibration method cannot ease ultra-wide bin problems caused by bins with a bin width $>2$ LSB. When dealing with ultra-wide bins (e.g., Bin [ $n]$ highlighted in blue in Fig. 2a), the mixed calibration method introduces missing codes, like Bin $[M+1]$ highlighted in red in Fig. 2a, and degrades the resolution. Moreover, the two-step mixed calibration method is unsuitable for automatic calibration. Therefore, we propose a new calibration method, the single-step weighted histogram calibration method. Unlike the mixed calibration method, the proposed method (Fig. 2b) can ease ultra-wide bin problems, detailed below.

Figure 3 shows the hardware implementation of the proposed weighted histogram calibration method. To achieve the histogramming function, the adders in Fig. 3 perform like accumulations. Like the mixed calibration method, the proposed method contains two parts (the bin compensation and width calibration). In the proposed method, three pairs of address factors ( $A d d r L, A d d r M$, and $A d d r R$ ) and width factors ( Coe L, Coe M, and Coe R) are stored in a calibration BRAM module, and the results are stored in three Histogram BRAM modules. There are three possible cases for bin compensation in the proposed calibration method, as shown in Figs 4a-c:

Case A: $W[k] \leq 1 \mathrm{LSB}$,

Case B: 1 LSB $<W[k] \leq 2$ LSB,

Case C: 2 LSB $<W[k] \leq 3$ LSB.

$W[k]$ is the bin width of the $k$-th actual bin. Figure 5 shows the pseudo-codes for calculating address factors. Ideally, with three pairs of factors, the proposed method can ease ultra-wide bin problems caused by bins whose width is less than 5 LSB. Figure $2 \mathrm{~b}$ is an example of this scenario: $\mathrm{Bin}[n]$ is the ultra-wide bin $(W[n] \leq 5$ LSB $)$ neighbored by two wide bins, Bin $[n-1]$ and $\operatorname{Bin}[n+1]$. Bin $[n]$ can be only remapped to three ideal bins (Bin $[M-1]$, Bin $[M]$, and Bin $[M+1])$. However, with the factors Addr $R[n-1]$ and $A d d r L[n+1]$, the ideal bins (Bin [M-2] and Bin $[M+2])$ are fulfilled by the actual bins (Bin $[n-1]$ and Bin $[n+1])$. Therefore, the ultra-wide bin problem caused by $\operatorname{Bin}[n]$ $(W[n] \leq 5 \mathrm{LSB})$ can be eased with the proposed method, which is more efficient than the mixed calibration method in [35].

Calculations for width factors follow similar rules shown in Fig. 4. In Case A, only Coe $L$ is valid and can be expressed as:

$$
\operatorname{Coe} L[k]=\frac{\text { Width }[k, n]}{W[k]},
$$

where Width $[k, n]$ is a portion of Bin $[k]$ in the actual TDL which should be in Bin $[n]$ in the ideal TDL (highlighted in red in Fig. 4a). 


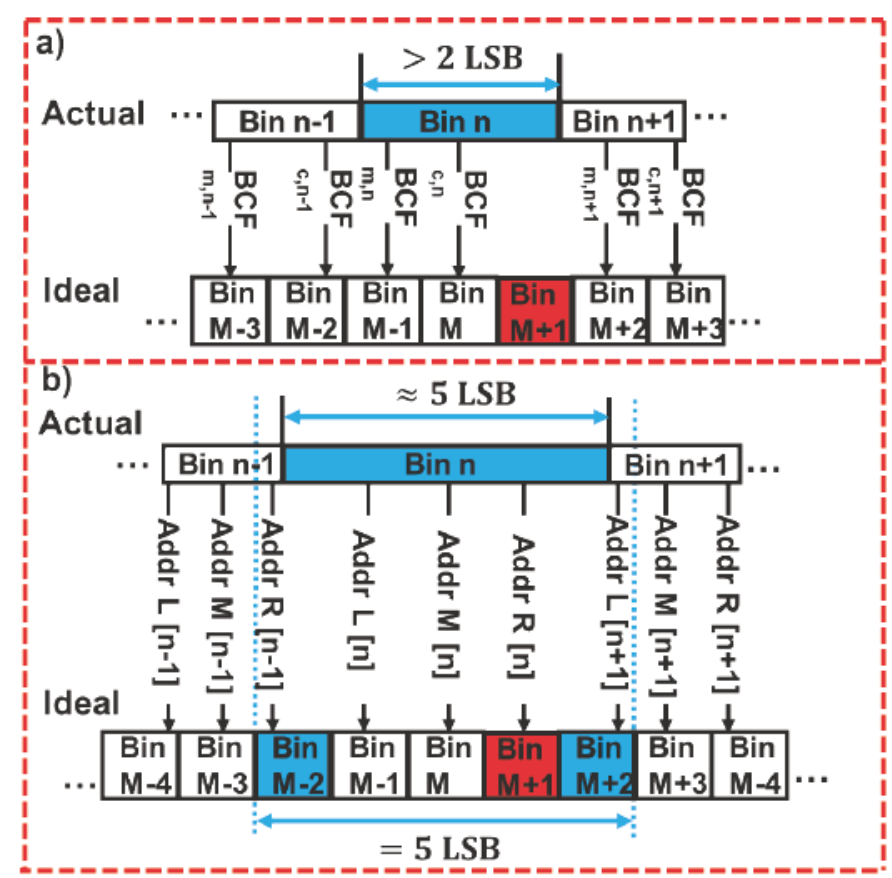

Fig. 2. Examples of bin compensation in a) the mixed calibration method [35] and b) the proposed weighted histogram calibration method.

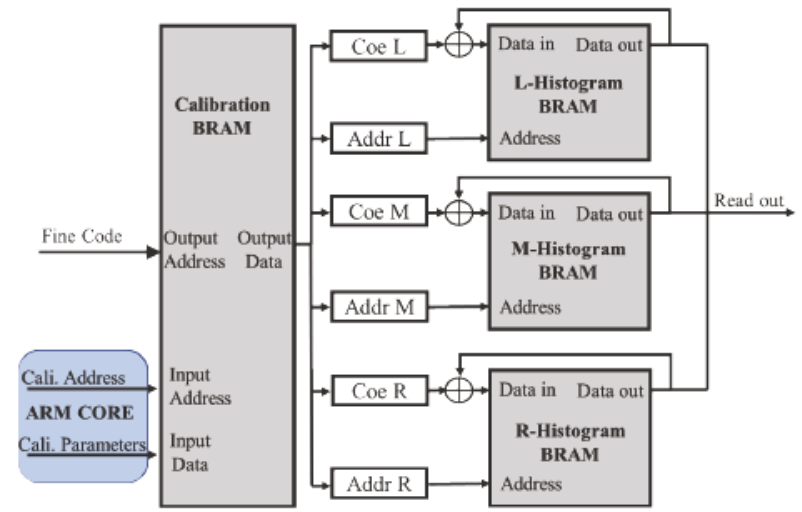

Fig. 3. Hardware implementation of weighted histogram calibration.

In Case B, Bin $[k]$ is remapped to two ideal bins (see Fig. $4 \mathrm{~b}$ ). Therefore, Coe $L$ and Coe $M$ can be defined as:

$$
\begin{gathered}
\text { Coe } L[k]=\frac{\text { Width }[k, n-1]}{W[k]}, \\
\text { Coe } M[k]=\frac{\text { Width }[k, n]}{W[k]},
\end{gathered}
$$

In Case $\mathrm{C}$, all width factors are valid and can be calculated as:

$$
\begin{gathered}
\text { Coe } L[k]=\frac{\text { Width }[k, n-1]}{W[k]}, \\
\text { Coe } M[k]=\frac{\text { Width }[k, n]}{W[k]}, \\
\text { Coe } R[k]=\frac{\text { Width }[k, n+1]}{W[k]} .
\end{gathered}
$$

Compared with the mixed calibration method in [35], which needs two-round code density tests, the proposed weighted histogram calibration method can calculate both address factors and width factors in a single round (see the difference between Fig. 6a and Fig. 6b, highlighted in yellow), which is more suitable for automatic calibration.

\section{Automatic Calibration}

The proposed AC function is based on Zynq SoC architectures. The workflow of the proposed function is shown in Fig. 6c and can be divided into two parts: the initial and the measurement stages. After powering up the board, the ARM core in PS calculates calibration factors and loads them to BRAMs in PL in the initial stage. In the measurement stage, indexed by raw data, the calibrated results will be delivered from the calibration module and stored in the histogramming module. Through this procedure, real-time and automatic calibration can be achieved with the ARM core and BRAM modules.

The system is free from manual or offline calibrations with the proposed automatic calibration function, suitable for broader commercial multichannel FPGA-TDC applications.

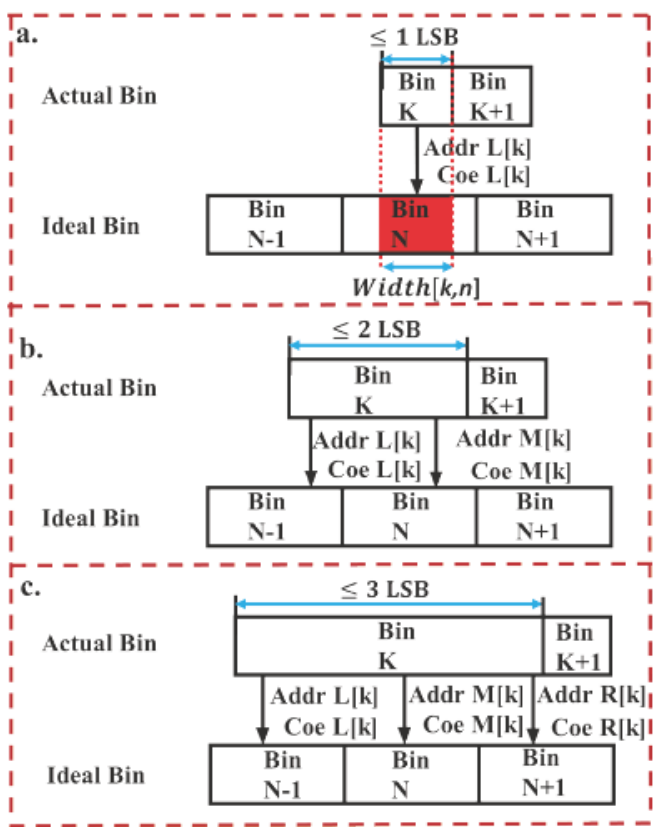

Fig. 4. Bin compensation when a) $\bar{W}[\bar{k}] \leq \overline{1} \overline{\mathrm{LSB}}, \overline{\mathrm{b}}) \overline{1} \mathrm{LSB}<\bar{W} \overline{[}] \leq 2$ LSB, and c) $2 \mathrm{LSB}<w[k] \leq 3 \mathrm{LSB}$.

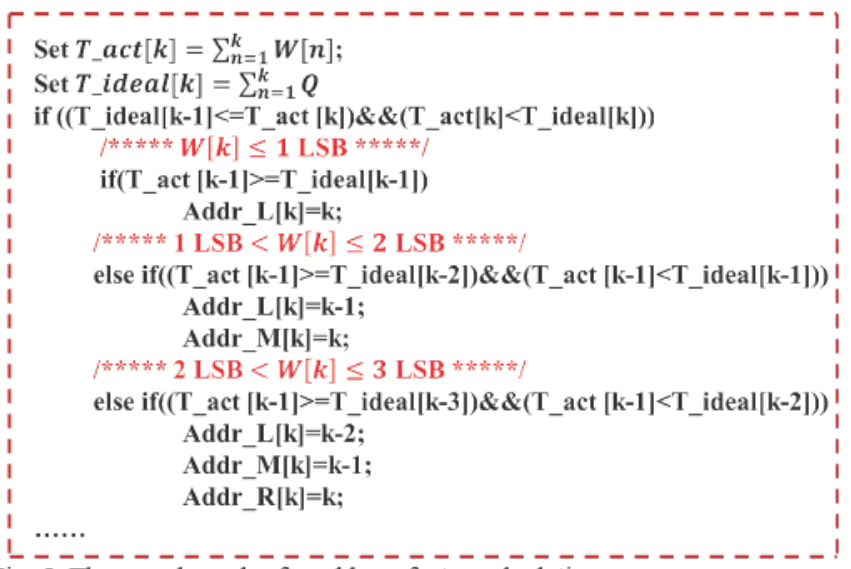

Fig. 5. The pseudo-codes for address factor calculations. 


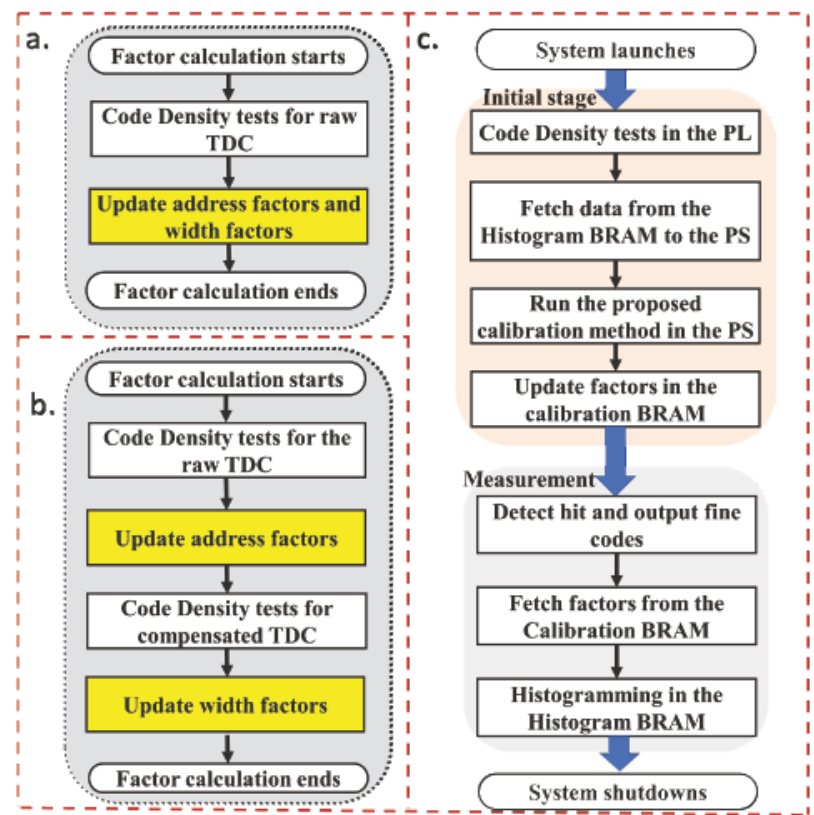

Fig. 6. Flow diagrams of (a) the proposed weighted histogram calibration method, (b) the mixed calibration method in [35]. (c) the workflow of AC TDCs.

\section{EXPERIMENTAL RESULTS}

We conducted experiments to evaluate the AC-WU TDC. Two asynchronous clocks from independent low-jitter crystal oscillators (Fox-767) were used as the system clock and the random input for code density tests. The temperature and the operation voltage were maintained in the experiment.

\section{A. Linearity and Bin Width Distribution}

The TDC's linearity is mainly evaluated by DNL, INL and their standard deviations ( $\sigma_{D N L}$ and $\sigma_{I N L}$ ). Besides, the equivalent bin-width $\omega_{e q}$ and its standard deviation $\sigma_{e q}$ were also proposed to evaluate TDC's linearity [51]. They are defined as:

$$
\sigma_{e q}^{2}=\sum_{i=1}^{N}\left(\frac{w[i]^{2}}{12} \times \frac{w[i]}{w_{\text {total }}}\right),
$$

where

$$
W_{\text {total }}=\sum_{i=1}^{N} W[i] \text {, }
$$

and

$$
\omega_{e q}=\sigma_{e q} \sqrt{12}=\sqrt{\sum_{i=1}^{n}\left(\frac{W[i]^{3}}{W_{\text {total }}}\right)} .
$$

The AC-WU TDC's DNL and INL are improved significantly compared with the uncalibrated TDC. The experimental results are summarized and shown in Table II and Fig. 7. After the calibration, $D N L_{p k-p k}$ and $I N L_{p k-p k}$ are improved by 13 -fold (from $3.91 \mathrm{LSB}$ to $0.30 \mathrm{LSB}$ ) and 18-fold (from $12.05 \mathrm{LSB}$ to $0.67 \mathrm{LSB}$ ), respectively. $\sigma_{D N L}$ is enhanced by 21 -fold (from $0.86 \mathrm{LSB}$ to $0.04 \mathrm{LSB}$ ), and $\sigma_{I N L}$ is enhanced by 21 -fold (from 2.79 LSB to $0.13 \mathrm{LSB}$ ). Besides, $\omega_{e q}$ and $\sigma_{e q}$ are improved from 19.76 ps to 9.85 ps and from 5.70 ps to 2.84 ps, respectively. Meanwhile, the AC-WU TDC's bin-width distribution is shown in Fig. 8a. The calibrated TDC shows a much more concentrated bin-width distribution than the uncalibrated TDC, as shown in Fig. 8b.

\section{B. Time Interval Tests}

The standard deviation of repeated measurements can evaluate the proposed TDC's RMS resolution. The built-in programmable delay modules, IDELAYE2 and IDELAYCTRL, can generate a controllable delay between the sampling clock and the hit signal [52]. Benefiting from the delayed signal generated inside the FPGA device, external measurement errors and jitters are minimized. As shown in Fig. 9a, we conducted 30 measurements covering one sampling clock, and each measurement captures $100 \quad 000$ samples. The standard deviations of each measurement were calculated, and the average value (13.86 ps) is the TDC's RMS resolution. Meanwhile, the measured histogram of a 980 ps time interval is shown in Fig. 9b, achieving 14.16 ps RMS resolution.

TABLE II

LINEARITY COMPARISON BETWEEN THE UNCALIBRATED TDC AND

\begin{tabular}{c|c|c}
\multicolumn{3}{c}{ CALIBRATED TDC } \\
\hline & $\begin{array}{c}\text { Tuned \& Sub- } \\
\text { WU }\end{array}$ & AC-WU \\
\hline LSB (ps) & \multicolumn{2}{|c}{$\mathbf{9 . 8 3}$} \\
\hline DNL (LSB) & {$[-0.93,2.98]$} & {$[-\mathbf{0 . 1 4 , 0 . 1 6}]$} \\
$D N L_{p k-p k}(\mathrm{LSB})$ & 3.91 & $\mathbf{0 . 3 0}$ \\
$\sigma_{D N L}(\mathrm{LSB})$ & 0.86 & $\mathbf{0 . 0 4}$ \\
INL (LSB) & {$[-6.52,5.53]$} & {$[-\mathbf{0 . 2 5 , 0 . 4 2}]$} \\
$I N L_{p k-p k}(\mathrm{LSB})$ & 12.05 & $\mathbf{0 . 6 7}$ \\
$\sigma_{I N L}(\mathrm{LSB})$ & 2.79 & $\mathbf{0 . 1 3}$ \\
$\omega_{e q}(\mathrm{ps})$ & 19.76 & $\mathbf{9 . 8 5}$ \\
$\sigma_{\boldsymbol{\theta} q}(p s)$ & 5.70 & $\mathbf{2 . 8 4}$ \\
\hline
\end{tabular}
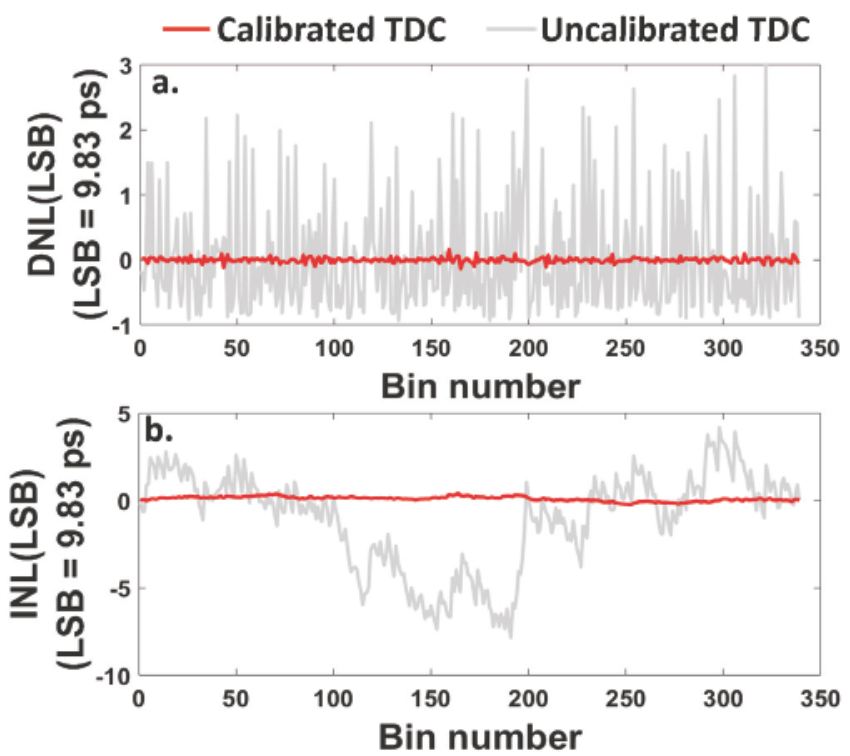

Fig.7 (a) DNL and (b) INL plots of the calibrated and uncalibrated TDCs.
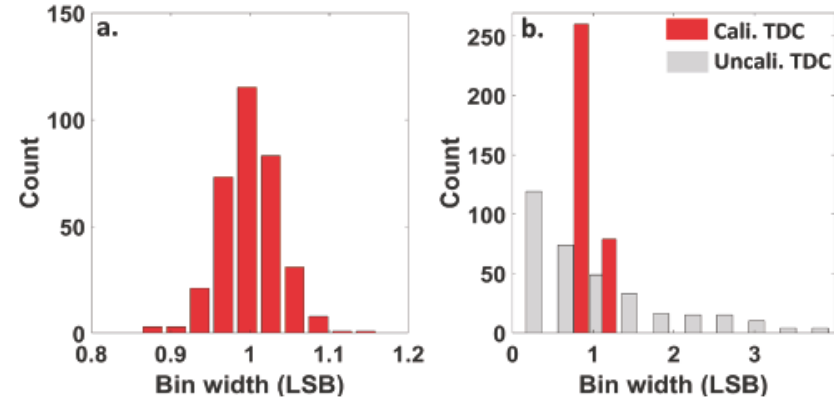

Fig. 8. Distribution of (a) calibrated bin-widths and (b) uncalibrated bin-widths. LSB $=9.83 \mathrm{ps}$. 


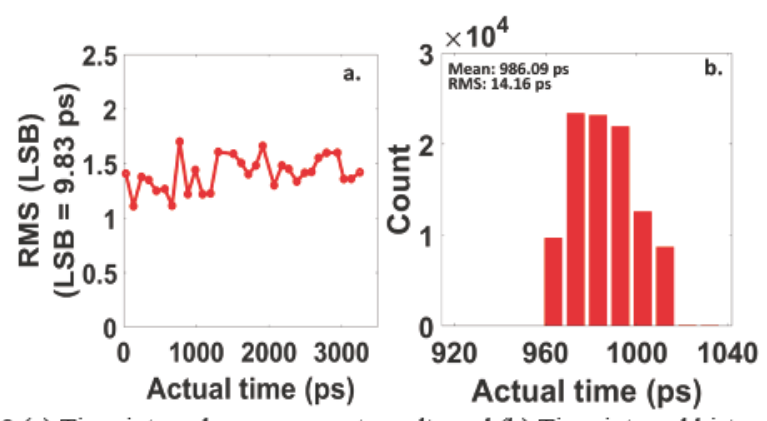

Fig. 9 (a) Time interval measurement results and (b) Time interval histogram at the time interval about $980 \mathrm{ps}$.
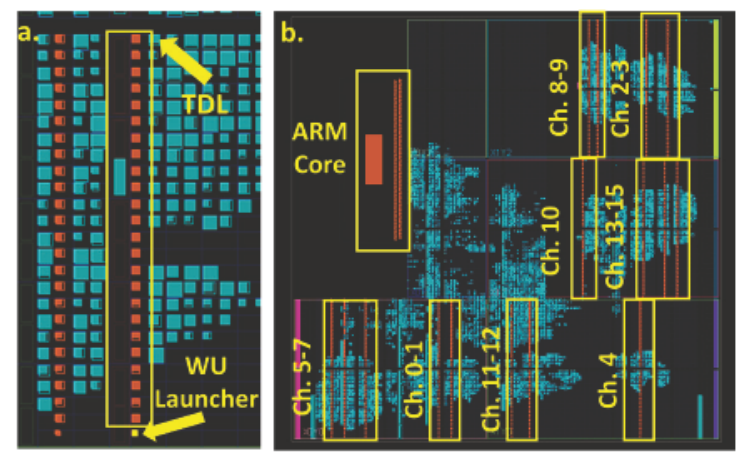

Fig. 10. Implementation layouts of (a) a single channel and (b) 16 channels.

Table III

Logic Resources Utilization

\begin{tabular}{c|cccc}
\hline & CARRY4 & LUTs & DFFs & BRAM \\
\hline Available & 13300 & 53200 & 106400 & 140 \\
\hline $\begin{array}{c}\text { Single- } \\
\text { channel }\end{array}$ & $\begin{array}{c}\mathbf{5 0} \\
(0.38 \%)\end{array}$ & $\begin{array}{c}\mathbf{7 6 4} \\
(1.44 \%)\end{array}$ & $\begin{array}{c}\mathbf{1 0 9 5} \\
(1.02 \%)\end{array}$ & $\begin{array}{c}\mathbf{2} \\
(1.42 \%)\end{array}$ \\
\hline 16-channel & $\begin{array}{c}\mathbf{8 0 0} \\
(6.02 \%)\end{array}$ & $\begin{array}{c}\mathbf{9 6 8 1} \\
(18.19 \%)\end{array}$ & $\begin{array}{c}\mathbf{1 5 1 4 1} \\
(14.23 \%)\end{array}$ & $\begin{array}{c}\mathbf{3 2} \\
(22.85 \%)\end{array}$ \\
\hline AXI Bus & $\mathbf{0}$ & $\begin{array}{c}\mathbf{7 9 7} \\
(1.49 \%)\end{array}$ & $\begin{array}{c}\mathbf{1 2 7 8} \\
(1.20 \%)\end{array}$ & $\mathbf{0}$ \\
\hline
\end{tabular}

\section{Multichannel Implementation and Logic Resources Consumption}

We have implemented and tested a 16-channel AC-WU TDC in Zynq-7000 SoCs. To avoid significant clock skews, each TDL (containing 50 CARRY4s) is placed within a clock region. Every WU launcher is constrained near the corresponding TDL's first CARRY4 to minimize jitters introduced by routing resources (see Fig. 10a). To show the efficiency of the proposed weighted histogram calibration method, 16 channels are distributed in the tested chip randomly (see Fig. 10b).

Table III summarizes the resource consumption for the 16channel design. Each channel costs 764 LUTs, 1095 DFFs, and 2 BRAMs. The usage report shows the proposed TDC architecture has great potential in multichannel applications. Moreover, the AXI bus is for communications between PL and PS with the cost of 1278 DFFs and 797 LUTs.
Code density tests were conducted for all channels. The linearity performances for 16 channels are concluded in Table IV, showing that the proposed 16-channel TDC has good uniformity.

\section{COMPARISON AND DISCUSSIONS}

Table V compares our proposed calibration method with other published calibration methods. Table VI summarizes recently published FPGA-TDCs and the proposed TDC.

Although calibration methods like bin-by-bin calibration [33], bin-width calibration [53], and mixed calibration [35] methods can improve linearity and precision, these methods require manual calibration, which is unsuitable for commercial products. The gain and error calibration [54] can correct data automatically, using signal processing methods (efficient but complex). However, this method [54] requires two signal interpolators and consumes more resources per channel than our solution (see Table VI).

Unlike ultra-high resolution ( $<5 \mathrm{ps)}$ TDCs [41], [47], [54][57], the AC-WU TDC aims to achieve a high resolution and linearity simultaneously in low-cost SoC devices. Compared with previously reported TDCs with similar resolutions, the proposed TDCs are easy to implement in modern SoC devices and have better linearity. Compared with the PLL delay matrix TDC proposed in [58], our TDC has similar linearity performances. However, the PLL delay matrix TDC requires 6fold more LUTs than our TDC with the AXI bus. Meanwhile, easy implementation is essential for broader applications. In this aspect, our design shows advantages compared with the two-stage delay line loop shrinking TDC [59] and the bidirectional RO Vernier TDC [60] because modern FPGAs do not have dedicated logic resources to construct loop architectures.

The proposed work allows researchers to produce repeatable measurements using established ARM-based ZYNQ devices easily. The proposed approach can also be implemented with open-source softcore processors for low-cost commercial developments. However, compared with the proposed TDC, the resources consumption of a softcore processor in FPGAs is close to or even higher than a single-channel design. They cost at least thousands of LUTs and DFFs [61], [62]. The Rocket Chip Generator [63], a dedicated open-source core, and its variation $S-R I S C-V$ [64] consume more than 30K LUTs and $15 \mathrm{~K}$ DFFs. Resource optimization in softcore processors is complex and requires expertise in implementing control buses and data buses and developing arithmetic logical units (ALUs).

Generally, benefiting from automatic calibration with ARMcore processors, our design does not require manual calibration like the direct histogram TDC [53] and the mixed calibration TDC [35]. This advantage widens the AC-WU TDC's applications.

Table IV

Summary of Linearity Performances of 16-Channel TDCs (Units: LSB)

\begin{tabular}{c|c|c|c|c|c|c|c|c|c|c|c|c|c|c|c|c|c|c|c|c|c|c|}
\hline Channel & 0 & 1 & 2 & 3 & 4 & 5 & 6 & 7 & 8 & 9 & 10 & 11 & 12 & 13 & 14 & 15 & Ave. \\
\hline$D N L_{p k-p k}$ & 0.21 & 0.34 & 0.33 & 0.28 & 0.30 & 0.29 & 0.56 & 0.69 & 0.25 & 0.41 & 0.25 & 0.26 & 0.52 & 0.27 & 0.48 & 0.60 & 0.38 \\
$\sigma_{D N L}$ & 0.03 & 0.05 & 0.05 & 0.04 & 0.04 & 0.04 & 0.10 & 0.07 & 0.04 & 0.06 & 0.03 & 0.04 & 0.07 & 0.03 & 0.06 & 0.07 & 0.05 \\
$I N L_{p k-p k}$ & 0.52 & 0.55 & 0.51 & 0.52 & 0.67 & 0.45 & 0.90 & 0.86 & 0.51 & 0.45 & 0.57 & 0.37 & 0.87 & 0.62 & 0.89 & 0.87 & 0.63 \\
$\sigma_{I N L}$ & 0.10 & 0.11 & 0.10 & 0.12 & 0.13 & 0.08 & 0.17 & 0.18 & 0.09 & 0.08 & 0.11 & 0.08 & 0.19 & 0.14 & 0.23 & 0.26 & 0.14 \\
\hline
\end{tabular}




\section{CONCLUSION}

We developed a multichannel auto-calibration architecture and a weighted histogram method for the first time. Besides, we combined the WU method, the tuned-TDL method and the subTDL method to implement high linearity, high resolution and multichannel TDCs in a low-cost ARM-based SoC device. The advantages are as follows.

1) Sometimes calibration methods require off-line or manual calibration (channel-by-channel or chip-by-chip). The proposed automatic calibration architecture resolves this problem by using ARM processors inside ARM-based SoC devices. The proposed TDC is more suitable for commercial products.

2) Traditional histogram-based calibration methods [35] need two steps to conduct address remapping and width calibration. We performed these two steps simultaneously for the proposed weighted histogram calibration method by analyzing relative positions between ideal and actual bins. Besides, the proposed method can ease ultra-wide bin problems caused by bins whose width is less than 5 LSB with only an extra BRAM module, compared with the mixed calibration method [35], which can only deal with 2 LSB ultra-wide bins.

Combining the WU method, the tuned-TDL method and the sub-TDL method, a novel TDC was proposed and evaluated. The bin size (LSB) achieves $9.83 \mathrm{ps}$, and $D N L_{p k-p k}$ and $I N L_{p k-p k}$ are enhanced to $0.38 \mathrm{LSB}$ and $0.63 \mathrm{LSB}$, respectively, in a low-cost Zynq-7000 ARM-based SoC. A 16-channel TDC system was also implemented and tested. The tests results show good uniformity between channels.

Table V

Comparison of published calibration methods

\begin{tabular}{c|ccc}
\hline $\begin{array}{c}\text { Ref-- } \\
\text { Year }\end{array}$ & Methods & $\begin{array}{c}\text { Multiple } \\
\text { steps }\end{array}$ & $\begin{array}{c}\text { Auto/manual } \\
\text { Calibration }\end{array}$ \\
\hline$[33]-10$ & Bin-by-bin calibration & Single-step & Manual \\
\hline$[53]-17$ & Bin-width calibration & Single-step & Manual \\
\hline$[35]-19$ & Mixed calibration & Two-step & Manual \\
\hline$[54]-21$ & Gain \& error calibration & Single-step & Auto \\
\hline $\begin{array}{c}\text { This } \\
\text { work }\end{array}$ & $\begin{array}{c}\text { Weighted histogram calibration } \\
\text { with an AC function }\end{array}$ & Single-step & Auto \\
\hline
\end{tabular}

Table VI

Comparison of Recently Published FPGA-TDCs

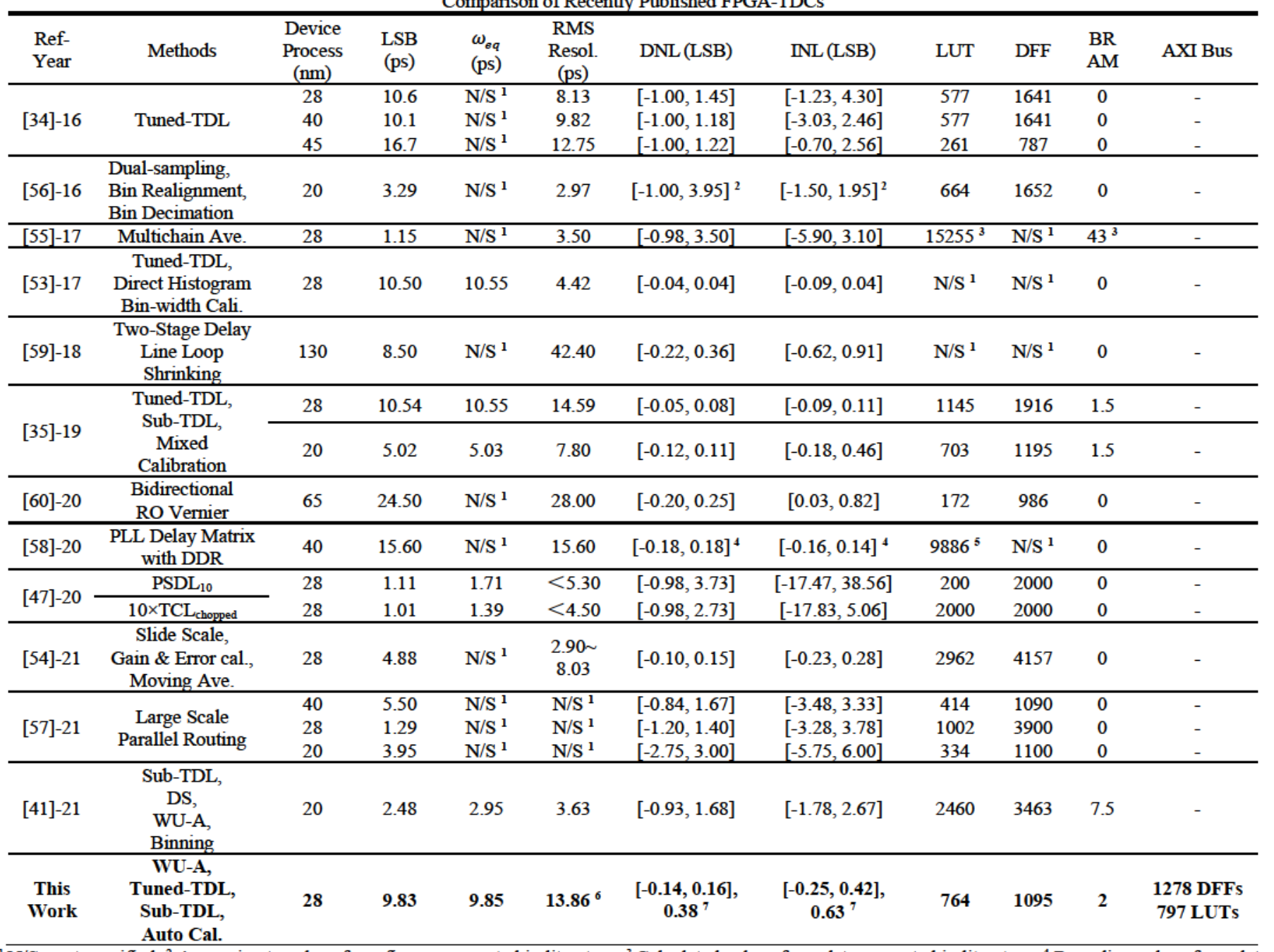

${ }^{1} \mathrm{~N} / \mathrm{S}=$ not specified $;{ }^{2}$ Approximate values from figures presented in literature; $;{ }^{3}$ Calculated values from data presented in literature; ${ }^{4}$ Rounding values from data presented in literature; ${ }^{5}$ Combinational ALUTs in Altera FPGA; ${ }^{6}$ The RMS resolution is measured internally. ${ }^{7}$ Averaged peak-peak DNL or INL results of the Multichannel TDCs. 
Previously published FPGA-TDCs were implemented in ARM-free FPGAs instead of ARM-based SoCs. The proposed architecture shows that ARM-based solutions can offer online automatic calibration over traditional FPGA devices, promising broader applications in LiDAR, PET-CT or Raman spectroscopy.

\section{ACKNOWLEDGEMENT}

The research has been supported by the Engineering and Physical Sciences Research Council under EPSRC Grant: EP/L01596X/1, the Royal Society of Edinburgh, and the China Scholarship Council. We would also like to acknowledge the support from Xilinx for donating FPGA development kits to the research group and the valuable input from Zhenya Zang regarding solutions using ARM devices and open-source softcore processors.

\section{REFERENCES}

[1] D. Li, M. Liu, R. Ma, and Z. Zhu, 'An 8-ch LIDAR Receiver Based on TDC With Multi-Interval Detection and Real-Time \$In Situ \$ Calibration', IEEE Trans. Instrum. Meas., vol. 69, no. 7, pp. 5081-5090, Jul. 2020.

[2] K. Kuzmenko et al., '3D LIDAR imaging using Ge-on-Si single\&\#x2013;photon avalanche diode detectors', Opt. Express, vol. 28, no. 2, pp. 1330-1344, Jan. 2020.

[3] S. Yang, B. Li, M. Liu, Y.-K. Lai, L. Kobbelt, and S.-M. Hu, 'HeteroFusion: Dense Scene Reconstruction Integrating Multi-Sensors', IEEE Trans. Vis. Comput. Graph., vol. 26, no. 11, pp. 3217-3230, Nov. 2020.

[4] Z. Zang, D. Xiao, and D. D.-U. Li, 'Non-fusion time-resolved depth image reconstruction using a highly efficient neural network architecture', Opt. Express, vol. 29, no. 13, pp. 19278-19291, Jun. 2021

[5] M. H. Nunes et al., 'Recovery of logged forest fragments in a humanmodified tropical landscape during the 2015-16 El Niño', Nat. Commun., vol. 12, no. 1, p. 1526, Dec. 2021.

[6] W. Pan, G. Gong, Q. Du, H. Li, and J. Li, 'High resolution distributed time-to-digital converter (TDC) in a White Rabbit network', Nucl. Instrum. Methods Phys. Res. Sect. Accel. Spectrometers Detect. Assoc. Equip., vol. 738, pp. 13-19, Feb. 2014.

[7] C.-C. Chen, C.-L. Chen, W. Fang, and Y.-C. Chu, 'All-Digital CMOS Time-to-Digital Converter With Temperature-Measuring Capability', IEEE Trans. Very Large Scale Integr. VLSI Syst., vol. 28, no. 9, pp. 2079-2083, Sep. 2020.

[8] X. Qin et al., 'An FPGA-Based Hardware Platform for the Control of Spin-Based Quantum Systems', IEEE Trans. Instrum. Meas., vol. 69, no. 4 , pp. $1127-1139$, Apr. 2020.

[9] W. Li et al., 'Time-tagged coincidence counting unit for large-scale photonic quantum computing', Rev. Sci. Instrum., vol. 89, no. 10, p. 103113 , Oct. 2018.

[10] M. Rudigier et al., 'FATIMA - FAst TIMing Array for DESPEC at FAIR', Nucl. Instrum. Methods Phys. Res. Sect. Accel. Spectrometers Detect. Assoc. Equip., vol. 969, p. 163967, Jul. 2020.

[11] M. Perenzoni, D. Perenzoni, and D. Stoppa, 'A 64 \$times\$ 64-Pixels Digital Silicon Photomultiplier Direct TOF Sensor With 100MPhotons/s/pixel Background Rejection and Imaging/Altimeter Mode With $0.14 \%$ Precision Up To $6 \mathrm{~km}$ for Spacecraft Navigation and Landing', IEEE J. Solid-State Circuits, vol. 52, no. 1, pp. 151-160, Jan. 2017.

[12] J. Lin, Y. Wang, Q. Cao, J. Kuang, and L. Wang, 'True random number generation based on arrival time and position of dark counts in a multichannel silicon photomultiplier', Rev. Sci. Instrum., vol. 90, no. 11, p. 114704, Nov. 2019.

[13] I Nissinen, J. Nissinen, P. Keranen, D. Stoppa, and J. Kostamovaara, 'A \$16times256\$ SPAD Line Detector With a 50-ps, 3-bit, 256Channel Time-to-Digital Converter for Raman Spectroscopy', IEEE Sens. J., vol. 18, no. 9, pp. 3789-3798, May 2018.

[14] C. Wang et al., 'A Real Time Coincidence System for High Count-Rate TOF or Non-TOF PET Cameras Using Hybrid Method Combining
AND-Logic and Time-Mark Technology', IEEE Trans. Nucl. Sci., vol. 57, no. 2, pp. 708-714, Apr. 2010

[15] S. Konugolu Venkata Sekar et al., 'Broadband Time Domain Diffuse Optical Reflectance Spectroscopy: A Review of Systems, Methods, and Applications', Appl. Sci., vol. 9, no. 24, p. 5465, Dec. 2019.

[16] F. M. D. Rocca et al., 'Real-time fluorescence lifetime actuation for cell sorting using a CMOS SPAD silicon photomultiplier', Opt. Lett., vol. 41, no. 4, p. 673, Feb. 2016.

[17] D. Xiao et al., 'Dynamic fluorescence lifetime sensing with CMOS single-photon avalanche diode arrays and deep learning processors', Biomed. Opt. Express, vol. 12, no. 6, pp. 3450-3462, Jun. 2021.

[18] R. Machado, J. Cabral, and F. S. Alves, 'Recent Developments and Challenges in FPGA-Based Time-to-Digital Converters', IEEE Trans. Instrum. Meas., vol. 68, no. 11, pp. 4205-4221, Nov. 2019.

[19] J. Sánchez-Garrido et al., 'A White Rabbit-Synchronized Accurate Time-Stamping Solution for the Small-Sized Cameras of the Cherenkov Telescope Array', IEEE Trans. Instrum. Meas., vol. 70, pp. $1-14,2021$.

[20] W. Zhang, R. Ma, X. Wang, H. Zheng, and Z. Zhu, 'A High Linearity TDC With a United-Reference Fractional Counter for LiDAR', IEEE Trans. Circuits Syst. Regul. Pap., 2021.

[21] H. Ma et al., 'On-Chip Trust Evaluation Utilizing TDC-Based Parameter-Adjustable Security Primitive', IEEE Trans. Comput.-Aided Des. Integr. Circuits Syst., pp. 1-1, 2020

[22] J. Kalisz, 'Review of methods for time interval measurements with picosecond resolution', Metrologia, vol. 41, no. 1, pp. 17-32, Feb. 2004.

[23] Swabian Instruments, 'Time Tagger Series - Brochure'. https://www.swabianinstruments.com/static/downloads/TimeTaggerSe ries.pdf.

[24] PicoQuant, 'PicoQuant Photon Counting and Timing'. https://www.picoquant.com/images/uploads/downloads/7304photon_counting_brochure.pdf.

[25] Becker \& Hickl GmbH, 'The bh TCSPC Handbook', Sep. 2019. https://www.becker-hickl.com/wp-content/uploads/2019/09/hb-bhTCSPC-1.pdf.

[26] 'ID900 Brochure', ID Quantique. https://marketing.idquantique.com/acton/attachment/11868/f-023e/1//-///-/ID900_Brochure.pdf (accessed Oct. 13, 2020).

[27] Xilinx, 'XST User Guide for Virtex-6, Spartan-6, and 7 Series Devices', Mar. 20, 2013. https://www.xilinx.com/support/documentation/sw_manuals/xilinx14_ 7/xst_v6s6.pdf.

[28] Xilinx, 'UltraScale Architecture Configurable Logic Block User Guide (UG574)'. 2017, Accessed: Nov. 05, 2019. [Online]. Available: https://www.xilinx.com/support/documentation/user_guides/ug574ultrascale-clb.pdf.

[29] J. Wu and Z. Shi, 'The 10-ps wave union TDC: Improving FPGA TDC resolution beyond its cell delay', in 2008 IEEE Nuclear Science Symposium Conference Record, Dresden, Germany, Oct. 2008, pp. 3440-3446

[30] Q. Shen et al., 'A 1.7 ps Equivalent Bin Size and 4.2 ps RMS FPGA TDC Based on Multichain Measurements Averaging Method', IEEE Trans. Nucl. Sci., vol. 62, no. 3, pp. 947-954, Jun. 2015.

[31] J. Kalisz, R. Szplet, R. Pelka, and A. Poniecki, 'Single-chip interpolating time counter with 200-ps resolution and 43-s range', IEEE Trans. Instrum. Meas., vol. 46, no. 4, pp. 851-856, Aug. 1997.

[32] A. M. Amiri, M. Boukadoum, and A. Khouas, 'A Multihit Time-toDigital Converter Architecture on FPGA', IEEE Trans. Instrum. Meas., vol. 58 , no. 3 , pp. 530-540, Mar. 2009

[33] J. Wu, 'Several Key Issues on Implementing Delay Line Based TDCs Using FPGAs', IEEE Trans. Nucl. Sci., vol. 57, no. 3, pp. 1543-1548, Jun. 2010.

[34] J. Y. Won and J. S. Lee, 'Time-to-Digital Converter Using a TunedDelay Line Evaluated in 28-, 40-, and 45-nm FPGAs', IEEE Trans. Instrum. Meas., vol. 65, no. 7, pp. 1678-1689, 2016.

[35] H. Chen and D. D.-U. Li, 'Multichannel, Low Nonlinearity Time-toDigital Converters Based on 20 and $28 \mathrm{~nm}$ FPGAs', IEEE Trans. Ind. Electron., vol. 66, no. 4, pp. 3265-3274, Apr. 2019.

[36] W. Xie, Y. Wang, H. Chen, and D. D.-U. Li, '128-channel highlinearity resolution-adjustable time-to-digital converters for LiDAR applications: software predictions and hardware implementations', IEEE Trans. Ind. Electron., pp. 1-1, 2021. 
[37] F. Garzetti, N. Corna, N. Lusardi, and A. Geraci, 'Time-to-Digital Converter IP-Core for FPGA at State of the Art', IEEE Access, vol. 9, pp. 85515-85528, 2021.

[38] S. Bourdeauducq, 'A 26 ps RMS time-to-digital converter core for Spartan-6 FPGAs', ArXiv13036840 Phys., Mar. 2013, Accessed: Aug. 08, 2021. [Online]. Available: http://arxiv.org/abs/1303.6840.

[39] Xilinx, 'Zynq-7000 SoC Data Sheet: Overview (DS190)', 2018. https://www.xilinx.com/support/documentation/data_sheets/ds190Zynq-7000-Overview.pdf (accessed Mar. 23, 2021).

[40] Xilinx, 'Zynq-7000 SoC and 7 Series Devices Memory Interface Solutions v4.2, User Guide (UG586)', 2018. https://www.xilinx.com/support/documentation/ip_documentation/mig _7series/v4_2/ug586_7Series_MIS.pdf (accessed Mar. 23, 2021).

[41] W. Xie, H. Chen, and D. D.-U. Li, 'Efficient time-to-digital converters in $20 \mathrm{~nm}$ FPGAs with wave union methods', IEEE Trans. Ind. Electron., pp. 1-1, 2021.

[42] Y. Wang, X. Zhou, Z. Song, J. Kuang, and Q. Cao, 'A 3.0-ps rms Precision 277-MSamples/s Throughput Time-to-Digital Converter Using Multi-Edge Encoding Scheme in a Kintex-7 FPGA', IEEE Trans. Nucl. Sci., vol. 66, no. 10, pp. 2275-2281, Oct. 2019.

[43] P. Kwiatkowski and R. Szplet, 'Multisampling wave union time-todigital converter', in 2020 6th International Conference on EventBased Control, Communication, and Signal Processing (EBCCSP), Krakow, Poland, Sep. 2020, pp. 1-5.

[44] Y. Wang and C. Liu, 'A Nonlinearity Minimization-Oriented ResourceSaving Time-to-Digital Converter Implemented in a $28 \mathrm{~nm}$ Xilinx FPGA', IEEE Trans. Nucl. Sci., vol. 62, no. 5, pp. 2003-2009, Oct. 2015.

[45] Z. Song, Y. Wang, and J. Kuang, 'A 256-channel, high throughput and precision time-to-digital converter with a decomposition encoding scheme in a Kintex-7 FPGA', J. Instrum., vol. 13, no. 05, pp. P05012P05012, May 2018.

[46] Y. Wang, J. Kuang, C. Liu, and Q. Cao, 'A 3.9-ps RMS Precision Timeto-Digital Converter Using Ones-Counter Encoding Scheme in a Kintex-7 FPGA', IEEE Trans. Nucl. Sci., vol. 64, no. 10, pp. 27132718 , Oct. 2017.

[47] P. Kwiatkowski and R. Szplet, 'Efficient Implementation of Multiple Time Coding Lines-Based TDC in an FPGA Device', IEEE Trans. Instrum. Meas., vol. 69, no. 10, pp. 7353-7364, Oct. 2020.

[48] N. Lusardi, F. Garzetti, and A. Geraci, 'The role of sub-interpolation for Delay-Line Time-to-Digital Converters in FPGA devices', Nucl. Instrum. Methods Phys. Res. Sect. Accel. Spectrometers Detect. Assoc. Equip., vol. 916, pp. 204-214, Feb. 2019.

[49] C. Liu and Y. Wang, 'A 128-Channel, 710 M Samples/Second, and Less Than 10 ps RMS Resolution Time-to-Digital Converter Implemented in a Kintex-7 FPGA', IEEE Trans. Nucl. Sci., vol. 62, no. 3, pp. 773-783, Jun. 2015.

[50] Xilinx, 'Xilinx 7 Series FPGA and Zynq-7000 All Programmable SoC Libraries Guide for HDL Designs (UG768)', 2013. https://www.xilinx.com/support/documentation/ip_documentation/mig _7series/v4_2/ug586_7Series_MIS.pdf (accessed Mar. 23, 2021).

[51] $\overline{\mathrm{J}} . \mathrm{Wu}$, 'Uneven bin width digitization and a timing calibration method using cascaded PLL', in 2014 19th IEEE-NPSS Real Time Conference, Nara, Japan, May 2014, pp. 1-4.

[52] Xilinx, '7 Series FPGAs SelectIO Resources User Guide (UG471)', 2018.

https://www.xilinx.com/support/documentation/user_guides/ug471_7 Series SelectIO.pdf.

[53] H. Chen, Y. Zhang, and D. D.-U. Li, 'A Low Nonlinearity, MissingCode Free Time-to-Digital Converter Based on 28-nm FPGAs With Embedded Bin-Width Calibrations', IEEE Trans. Instrum. Meas., vol. 66, no. 7, pp. 1912-1921, Jul. 2017.

[54] K.-J. Choi and D.-W. Jee, 'Design and Calibration Techniques for a Multichannel FPGA-Based Time-to-Digital Converter in an Object Positioning System', IEEE Trans. Instrum. Meas., vol. 70, pp. 1-9, 2021.

[55] X. Qin, L. Wang, D. Liu, Y. Zhao, X. Rong, and J. Du, 'A 1.15-ps Bin Size and 3.5-ps Single-Shot Precision Time-to-Digital Converter With On-Board Offset Correction in an FPGA', IEEE Trans. Nucl. Sci., vol. 64, no. 12, pp. 2951-2957, Dec. 2017.

[56] Y. Wang and C. Liu, 'A 4.2 ps Time-Interval RMS Resolution Timeto-Digital Converter Using a Bin Decimation Method in an UltraScale FPGA', IEEE Trans. Nucl. Sci., vol. 63, no. 5, pp. 2632-2638, Oct. 2016.
[57] M. Zhang, K. Yang, Z. Chai, H. Wang, Z. Ding, and W. Bao, 'HighResolution Time-to-Digital Converters Implemented on 40-, 28-, and 20-nm FPGAs', IEEE Trans. Instrum. Meas., vol. 70, pp. 1-10, 2021.

[58] P. Chen et al., 'High-Precision PLL Delay Matrix With Overclocking and Double Data Rate for Accurate FPGA Time-to-Digital Converters', IEEE Trans. Very Large Scale Integr. VLSI Syst., vol. 28, no. 4, pp. 904-913, Apr. 2020.

[59] J. Zhang and D. Zhou, 'An 8.5-ps Two-Stage Vernier Delay-Line Loop Shrinking Time-to-Digital Converter in 130-nm Flash FPGA', IEEE Trans. Instrum. Meas., vol. 67, no. 2, pp. 406-414, Feb. 2018.

[60] K. Cui and X. Li, 'A High-Linearity Vernier Time-to-Digital Converter on FPGAs With Improved Resolution Using Bidirectional-Operating Vernier Delay Lines', IEEE Trans. Instrum. Meas., vol. 69, no. 8, pp. 5941-5949, Aug. 2020.

[61] Á. B. de Oliveira et al., 'Evaluating Soft Core RISC-V Processor in SRAM-Based FPGA Under Radiation Effects', IEEE Trans. Nucl. Sci., vol. 67 , no. 7 , pp. 1503-1510, Jul. 2020.

[62] Z. Zang, Y. Liu, and R. C. C. Cheung, 'Reconfigurable RISC-V Secure Processor And SoC Integration', in 2019 IEEE International Conference on Industrial Technology (ICIT), Feb. 2019, pp. 827-832.

[63] K. Asanovic et al., 'The Rocket Chip Generator'. [Online]. Available: https://www2.eecs.berkeley.edu/Pubs/TechRpts/2016/EECS-201617.html.

[64] Y. Liu, R. C. C. Cheung, and H. Wong, 'Lightweight Secure Processor Prototype on FPGA', in 2018 28th International Conference on Field Programmable Logic and Applications (FPL), Aug. 2018, pp. 4434431.

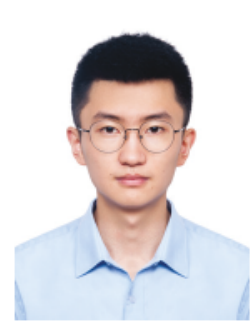

Yu Wang was born in Chongqing, China in 1995. He received the $B$.Eng. degree in measurement and control from the Harbin University of Science and Technology, in 2013, and the M.Eng. degree in electronics and communication engineering from Harbin Engineering University, in 2020. Since October 2020, he has been working toward the $\mathrm{Ph} . \mathrm{D}$. degree founded by China Scholarship Council at the University of Strathclyde, Glasgow, U.K. His current research interests include FPGA-based mixed signal circuits.

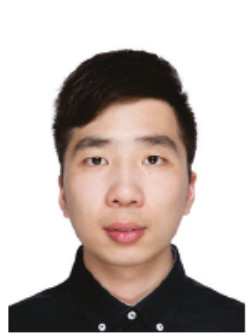

Wujun Xie was born in Hunan, China, in 1996. He received the B.Eng. degree in electronic and information engineering from the Hangzhou Dianzi University, Hangzhou, China, in 2017, and the M.S. degree in embedded systems from the University of Southampton, Southampton, U.K., in 2018. Since October 2018, he has been working toward the $\mathrm{Ph} . \mathrm{D}$. degree at the University of Strathclyde, Glasgow, U.K. His current research interests include high-precision timing circuits for time-resolved measurements in field-programmable-gate-array technology.

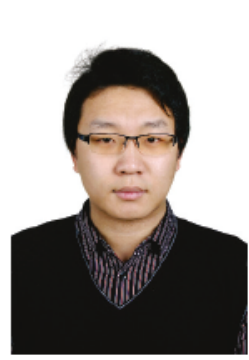

Haochang Chen was born in Shanxi, China, in 1990. $\mathrm{He}$ received the B.Sc degree in electronic design automation from the University of Central Lancashire, Preston, U.K., in 2012, the B.Eng. degree in electronic and information engineering from the North China University of Technology, Beijing, China, in 2012, the M.S. degree in embedded digital systems from the University of Sussex, Brighton, U.K., in 2013, and the $\mathrm{PhD}$ degree from the University of Strathclyde, Glasgow, U.K., in 2020, funded by EPSRC, and then he joined the Fraunhofer UK Research Ltd as a researcher. His current research interests include FPGA-based high-precision time metrology systems for ranging and biomedical imaging applications. 


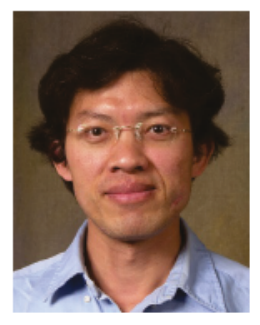

David Day-Uei $\mathrm{Li}$ received the $\mathrm{Ph} . \mathrm{D}$. degree in electrical engineering from the National Taiwan University, Taipei, Taiwan, in 2001. He then joined the Industrial Technology Research Institute, Taiwan, working on CMOS optical and wireless communication chipsets. From 2007 to 2011, he worked at the University of Edinburgh, Edinburgh, on two European projects focusing on CMOS singlephoton avalanche diode sensors and systems. He then took the lectureship in biomedical engineering at the University of Sussex, Brighton, in mid-2011, and in 2014 he joined the University of Strathclyde, Glasgow, as a Senior Lecturer. He has authored more than 90 journal and conference papers and holds 12 patents. His research interests include CMOS sensors and systems, mixed signal circuits, embedded systems, optical communications, FLIM systems and analysis, fieldprogrammable gate array computing, and machine-learning. His research exploits advanced sensor technologies to reveal low-light but fast biological phenomena. 